\title{
Probing nonlinearity of semi-macroscopic vacuum by second harmonic generation with intense laser fields
}

\author{
Kensuke Homma \\ Graduate School of Science, Hiroshima University, Kagamiyama, Higashi-Hiroshima 739-8526, \\ Japan, and Fakultät für Physik, Ludwig Maximilians Universität München, D-85748 Garching, \\ Germany
}

DOI: http://dx.doi.org/10.5689/UA-PROC-2010-09/44

\begin{abstract}
Astronomical observations suggest the presence of dark matter and dark energy. This may be attributable to undiscovered fields of small mass below $1 \mathrm{eV}[1,2,3]$. These fields are thought to evade our effort to detect them in laboratory, because they are supposed to weakly couple to matter. The high intense laser fields may open up a new window for probing these fields via the coupling to laser photons with its degenerated feature and huge statistics per pulse. In this talk I present a novel method to search for these fields by observing second harmonic generation of photons in the quasi-parallel colliding system by focusing a single laser beam $[4,5]$.
\end{abstract}

\section{Introduction}

Let us point out how little we observed various aspects of vacuum in different scales so far. What is shown in Fig. 1 is the system size of vacuum including matter states as a function of their energy densities. As the largest size, we can take the horizon size of the Universe. On the other hand, the extremely high energy density state can be obtained by $e^{+} e^{-}$colllider where many particle pairs are produced from the point-like high energy density system. We should notice that there is a huge vacant domain in this plot. Why is it so? This may be simply due to a fact that observational technologies limit the domains we can reach. After telescope has been invented in 16 century, we start observing phenomena happening in distant stars. On the other hand, once Rutherford invented a way to introduce high beam momentum to probe the microscopic structure inside an atom, we have begun to develop accelerators and the colliders to further probe smaller structures. Eventually we discovered many new phenomena and established the particle standard model. Therefore, if we could invent a new way to probe the vacant domain, we have many chances to discover something fundamentally new. Let us introduce another view point here. The reason why we needed massive and huge objects such as starts to discover gravitational force was because the coupling to matter is extremely small and the gravitational field can be exchanged over infinite distance, i.e., the mass of the exchanged field is zero. On the other hand what we can discuss in the extremely high energy density states is the limit of relatively strong coupling compared to the gravitational one by exchanging massive fields characterizing the short range interactions. In order to produce such heavier 


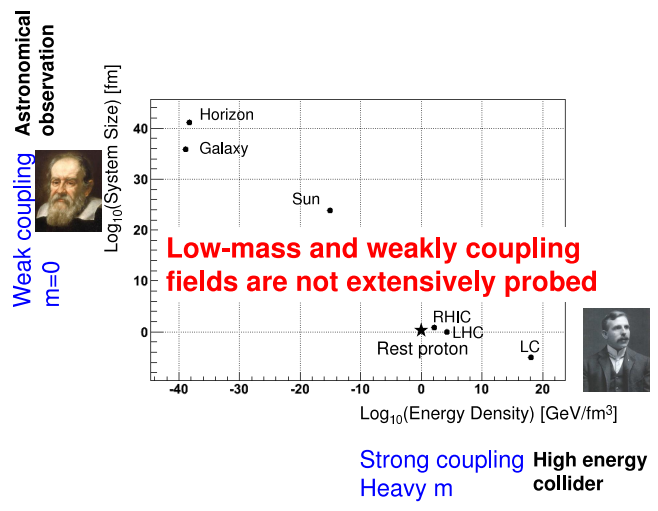

Figure 1: Experimental domains of various approaches to probe matter and vacuum as a function of the system size vs. the energy density. Selected systems are LC: electron-positron collision in the center-of-mass energy $E_{c m s}=1 \mathrm{TeV}$ at the future linear collider [6], assuming the electron size $10^{-18} \mathrm{~cm}$; LHC: proton-proton collision in $E_{c m}=14 \mathrm{TeV}$ at the Large Hadron Collider [6]; RHIC: gold-gold collision in $E_{c m}=200 \mathrm{GeV}$ per nucleon pair at the Relativistic Heavy Ion Collider [6], the rest proton indicated by the asterisk as the origin of this plot; Sun, the Milky Way Galaxy and the event horizon with $\Omega_{t o t} \sim 1.0$ and $h \equiv H_{0} / 100[\mathrm{~km} / \mathrm{s} / \mathrm{Mpc}] \sim 0.7[7]$.

masses, we were forced to increase the incident momentum scale in high energy accelerators. In this sense, the weakly coupling and low-mass particles are not extensively probed yet. In this talk, we suggest an approach to provide a new type of semi-macroscopic vacuum scope dedicated for this unproved domain by utilizing photon-photon interactions within high intense laser fields.

\section{Why photons?}

Space-time scales of phenomena are very important in physics. The scales between particle physics (field theory) and cosmology (geometry) are totally different, which may lead to the inconsistent picture on the definition of vacuum state as represented by dark energy problem. The observed dark energy density is too small to explain by simple field theoretical view point [8]. Therefore, it is important for experiments to collect information in different space-time scales as much as possible. In this respect photon is a valuable probe to see natures of vacuum, since we can introduce different frequencies by many orders of magnitude in laboratory experiments. If we could introduce $100 \mathrm{GeV}$ scale, we may be able to prove higgs field which is a scalar field to give masses to elementary particles. If we introduce $0.1 \mathrm{MeV}-100 \mathrm{MeV}$ scale, we can discuss photon-photon interactions through virtual vacuum polarizations by electron-positron and quark-antiquark loops. If we could provide photon energy well bellow $1 \mathrm{eV}$ scale, we may have chances to observe something undiscovered which are expected to be relevant for dark matter and dark energy. As an example that may be relevant for dark energy, let us remind you of the past experiments to probe non-Newtonian gravity [9]. In these experiments huge and massive bodies were used to measure a tiny deviation from the Newtonian potential form due 
to contamination of a finite range forth. However, if the distance between two bodies is short, these experiments suffered from the huge background force by Coulomb interactions. On the other hand, if we could use photons as the replacement of the massive test bodies, known background interactions are very much suppressed due to the small cross section of photon-photon scattering via virtual polarizations mentioned above in optical wave length [10]. However, the biggest issue appears, because the huge and massive probes were actually necessary to have a sensitivity to the gravitational coupling strength. Nevertheless, if we could overcome this drawback to use photons as the test probe, we may be able to develop a new method to probe weakly coupling and low-mass fields (finite long range force). As long as the field has a finite mass and coupling to matter, in principle, we can directly produce low-mass fields as resonance states such as higgs particle in high energy colliders. The production cross section is in principle free from the constraints by the weak coupling, if the center of mass system energy $E_{c m s}$ of two colliding photons is adjusted to the top of the resonance function.

\section{Quasi-Parallel System of photon-photon collisions}

If we are interested in extremely low-mass ranges below meV, we have to reduce the CMS energy of photon-photon collisions compared to incident photon energy if optical laser fields are assumed. As long as the resonance is allowed to decay into only two photons, the scattering process looks like an elastic scattering even if a low-mass field is exchanged via the resonance state in CMS. Thus there is no frequency shift in the final state in CMS. However, if we boost this system to the direction perpendicular to the colliding axis, the frequency shift takes place along that boosted axis. In the forward direction on the boost axis we expect frequency up shift to close to the double of the incident frequency, while zero frequency photon must be emitted to the backward direction due to the energy-momentum conservation independent of the dynamics of the exchanged field. This may be interpreted as if the second harmonic photon is generated from the nonlinear vacuum response. This could be an interesting analogy to the second harmonic generation due to the nonlinear response of a crystal with a laser injection which was pioneered by Franken et al.[11]. Inversely if we realize the QPS as a laboratory frame, the corresponding CMS energy can be very much lowered. The CMS energy in QPS can be defined as $E_{c m s} \sim 2 \vartheta \omega$ where $\vartheta$ is defined as a half incident angle between two incoming photons and $\omega$ is the beam energy in unit of $\hbar=c=1$. This relation indicates that we have two experimental handles to adjust $E_{c m s}$. If we take the head-on collision geometry, we have to introduce very long wavelength as the incident photons. However, it is not too difficult to introduce the very small incident angle. In such a case the $E_{c m s}$ can be lowered by keeping $\omega$ constant. We also know that the cross section of photo-photon scattering in QED process $\sigma_{q e d}$ in QPS is quite suppressed due to the fourth power dependence on the incident angle which is expressed as $\sigma_{q e d} \sim\left(\alpha^{2} / m_{e}^{4}\right)^{2} \omega^{6} \vartheta^{4}$ with the fine structure constant $\alpha$ and electron mass $m_{e}$ [12]. Therefore, the low frequency photons in QPS is the best system to probe such a low-mass field.

\section{QPS by focusing with single Gaussian laser beam}

However, it is difficult to introduce two colliding photon beams which satisfy the small incident angle based on the simple geometrical optics due to the wavy nature of photons in the diffraction limit of photons. Below meV range we are naturally led to introduce a geometry by focusing a single laser beam as illustrated in Fig.2. What is important here is that in the diffraction 


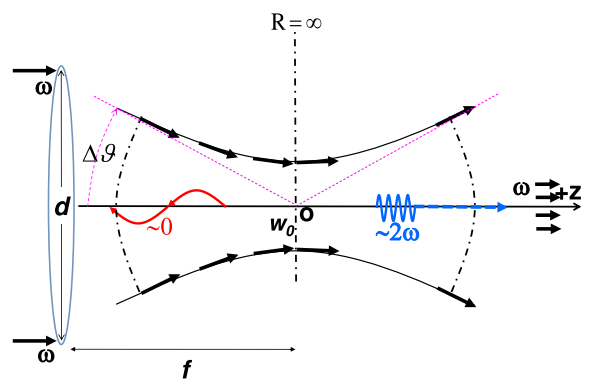

Figure 2: Second harmonic generation in Quasi Parallel System by focusing a single Gaussian laser beam.

limit there are uncertainties on the incident momentum due to uncertainty principle, in other words, there are uncertainties on the incident angles between two photons among the single beam, even though photons are in the degenerated state in the output of the laser crystal. This should be contrasted to the case of high energy collider where the momentum spread of each colliding particle or uncertainty based on the de Broglie length is negligibly small compared to the magnitudes of relevant momentum exchanges they are interested in. This different initial condition becomes critically important for the following discussions.

\section{Dynamics of photon-photon scattering}

As the simplest coupling between two photons and a low-mass field we focus on the quantum anomaly type coupling $g^{2} / M$ which includes square of electric charge $g$ to couple virtual fermion loops to two external photons and a dimensional coupling $1 / M$ to low-mass neutral fields[4]. If $M$ is Planckian mass scale of $10^{27} \mathrm{eV}$, the coupling expresses gravitational one. We may discuss possibilities to exchange scalar and pseudoscalar type of fields by requiring combinations of photon polarizations in the initial and final states [5]. The virtue of laser experiments is in the specifications on the all photon spin states both in the initial and final states in the two body photon-photon interaction. This allows us to discuss types of exchanged fields in general.

\section{How to overcome the extremely narrow resonance}

The exact resonance condition is the requirement of $m=E_{c m s}$ where $m$ is the mass of exchanged field. The squire of the scattering amplitude $A$ can be expressed as Breit-Wigner(BW) resonance function $|A|^{2}=(4 \pi)^{2} a^{2} /\left(\chi^{2}(\vartheta)+a^{2}\right)$ as a function of $\chi$ and width $a$ which are defined as $\chi(\vartheta) \equiv \omega^{2}-\omega_{r}^{2}(\vartheta)$ and $a \equiv\left(\omega_{r}^{2} / 16 \pi\right)\left(g^{2} m / M\right)^{2}$, respectively. The energy $\omega_{r}$ satisfying the resonance condition can be defined as $\omega_{r} \equiv m^{2} /\left(1-\cos 2 \vartheta_{r}\right)$ [5]. If we take Planckian mass as $M$, the width $a$ becomes extremely small. This implies that the resonance width is too small to hit the peak position of the resonance function. How can we overcome this problem?

We take a different approach to this problem. Let us remind you of higgs hunting by high energy colliders as an example to hit the top of the resonance. In high energy colliders the 
spread of the beam energy is much smaller than the width of the resonance function which they try to probe. On the hand, in collisions at the diffraction limit in QPS, the resonance width looks almost like delta-function and the uncertainty on the $E_{c m s}$ is much wider than the resonance width as we discussed in section 4 . We may use the following feature of deltafunction. Although the width of the delta-function is infinitesimal, as far as it is integrated over $\pm \infty$, the value of integral becomes order of unity. In the case of BW function, even if we integrate it over $\pm a$, the value of the integral is just a half of the value integrated over $\pm \infty$. This implies that as long as we capture the resonance peak within a finite beyond $\pm a$, the value of the integral becomes proportional to $a$ but not $a^{2}$. Actually in the diffraction limit incident angles of incoming photons are in principle uncertain. Thus we must use the averaged cross section by integrating the square of the scattering amplitude over possible range on $E_{c m s}$ determined by uncertainties on incident angles.

Let us reflect this feature in the case of single beam focusing experiment. We can introduce a probability distribution function on the possible incident angles between randomly selected photon pairs among the incident single laser beam. We then define the range of integral on the incident angel as $\Delta \vartheta$ based on the focusing parameter such as beam diameter and focal length. If this range does not contain the resonance angle $\vartheta_{r} \equiv m / 2 \omega$, that is, $\vartheta_{r}>\Delta \vartheta,|\bar{A}|^{2}$ becomes proportional to $a^{2}$ which indicates the suppression by $M^{-4}$. On the other hand, if $\vartheta_{r}<\Delta \vartheta$ is satisfied, we can obtain the proportionality to $a$, namely, sensitivity enhancement by $M^{2}$ compared to the case without resonance. Thus various focusing parameters to adjust $\Delta \vartheta$ by controlling the beam diameter and focal length can introduce a sharp cutoff on the cross section which eventually controls sensitive mass ranges of this method through the relation $m<2 \omega \Delta \vartheta$.

\section{Can we reach gravitational coupling strength?}

Let us now discuss how much intense laser fields are necessary to have a sensitivity to gravitational coupling strength based on the concept of photon-photon scattering in QPS. As the most challenging case let us assume $m \sim 10^{-9} \mathrm{eV}$ which can be a candidate of dark energy if it could couple to matter as weak as gravitational coupling strength $1 / M_{P} \sim 10^{-27} \mathrm{eV}^{-1}$ [3]. The yield of harmonic generation $\mathcal{Y}$ after integrated over the solid angle which satisfies the condition that one of final state photons has the frequency close to $2 \omega$, can be expressed as $\mathcal{Y} \sim\left(\vartheta_{r} / \Delta \vartheta\right)\left(g^{2} m / M\right)^{2} \sin ^{-2} \vartheta \mathcal{L}$, where $\left(\vartheta_{r} / \Delta \vartheta\right)$ is relevant for the normalization of the probability on incident angles, $\left(g^{2} \mathrm{~m} / M\right)^{2} \sim(\alpha \mathrm{m} / M)^{2} \sim 10^{-76}$ is the factor after averaging on BW resonance function, $\sin ^{-2} \vartheta$ is the factor related with the phase volume integral and the photon flux factor after integrated over the solid angle, and $\mathcal{L}$ is luminosity-like factor in the collider concept. The significant difference of $\mathcal{L}$ from that of collider concept is that photons are annihilated and created from degenerated states rather than from vacuum state. This implies that annihilation and creation operators cause $\sqrt{\bar{N}}$ in each vertex of the Feynman diagram with the mean number of photons $\bar{N}$ in a laser field by assuming $\bar{N}$ is large enough. On the production vertex of the low-mass field, we expect a factor of $\sqrt{\bar{N}}^{2}$ at the amplitude level due to two photon annihilation in the degenerated number state. Taking square of this factor gives a similar factor to collider luminosity which includes a factor proportinal to $n^{2}$ where $n$ is the number of charged particles per beam bunch. In order to have one second harmonic photon per laser focusing, we would need $10^{29}$ optical photons corresponding to $\sim 10^{11} \mathrm{~J}$ by assuming $\left(\vartheta_{r} / \Delta \vartheta\right) \sim 1$ with long focal length, though it might be too optimistic to realize it as terrestrial experiments. Furthermore, we may also induce decays of the produced resonances by adding a 
degenerated photon states with different frequency to let the one of two photons from the resonance decay into the prepared degenerated state, where creation operator causes the additional factor of $\sqrt{\bar{N}}$ at the scattering amplitude level. Therefore, if we mix two frequencies in advance with equal intensity $\bar{N}$, we may expect the increase of the luminosity-like factor $\mathcal{L} \sim \bar{N}^{3}$. In this case the necessary photon intensity may be very much reduced to $10^{19}$ optical photons corresponding to $\sim 10 \mathrm{~J}$ if $\left(\vartheta_{r} / \Delta \vartheta\right) \sim 1$ is assumed. We note that the photon frequency to be observed changes from the second harmonic of the incident photons depending on the frequency of the field to induce decays.

\section{Summary}

Higher harmonic generation in Quasi-Parallel-System can be a novel probe to discuss weakly coupling low-mass fields. Experimental realization of QPS as parallel as possible is a challenge for future experiments. A degenerated field to induce decay of resonance is important to probe coupling as weak as gravity though higher harmonic generation. Given high intensity laser fields $\sim 2 \mathrm{~kJ}$ per pulse available in Extreme Light Infrastructure[13], we foresee the breakthrough on the existing sensitivity to coupling of low-mass fields to photons based on this novel idea.

\section{Acknowledgments}

This work is based on intensive discussions with Y. Fujii, D. Habs and T. Tajima under support by the DFG Cluster of Excellence MAP (Munich-Center for Advanced Photonics). K.Homma acknowledges for support by the Grant-in-Aid for Scientific Research no.21654035 from MEXT of Japan in part.

\section{References}

[1] For example, see Figure 2 in J. Jaeckel and A. Ringwald, arXiv:1002.0329 [hep-ph].

[2] Y. F. Cai, E. N. Saridakis, M. R. Setare and J. Q. Xia, arXiv:0909.2776 [hep-th]; S. Tsujikawa, arXiv:1004.1493 [astro-ph.CO].

[3] Y. Fujii and K. Maeda, The Scalar-Tensor Theory of Gravitation (Cambridge Univ. Press, 2003).

[4] Y. Fujii and K. Homma, arXiv:1006.1762 [gr-qc].

[5] K. Homma, D. Habs and T. Tajima, arXiv:1006.4533 [quant-ph].

[6] See section for High-energy collider parameters in C. Amsler et al. (Particle Data Group), Phy. Lett. B667, 1 (2008) and 2009 partial update for the 2010 edition.

[7] D. N. Spergel et al., Astrophysical Journal Supplement, 148, 175 (2004)

[8] V. Sahni and A. A. Starobinsky, Int. J. Mod. Phys. D 9, 373 (2000) [arXiv:astro-ph/9904398].

[9] See, for example, Figures; 2.13, 4.16-17 in E. Fischbach and C. Talmadge, The Search for Non-Newtonian Gravity (AIP Press, Springer-Verlag, N.Y., 1998).

[10] B. De Tollis, Nuovo Cimento 32757 (1964); B. De Tollis, Nouvo Cimento 351182 (1965).

[11] P. Franken, A. E. Hill, C. W. Peters, and G. Weinreich, Phys. Rev. Lett. 7, 118 (1961). 36

[12] See p.183 in W. Dittrich and H. Gies, Probing the Quantum Vacuum (Springer, Berlin, 2007).

[13] http://www.extreme-light-infrastructure.eu/. 